\title{
Prevalencia de mansonelosis en comunidades del río Atabapo del departamento de Guanía, Colombia
}

\author{
Herbert Iván Vera ', Julián Trujillo ${ }^{2}$
}

\begin{abstract}
Resumen
En el departamento de Guainía, Colombia, se realizó un estudio tipo encuesta de prevalencia de corte en siete comunidades del río Atabapo, donde se calculó una muestra poblacional de 181 personas mayores de 10 años que se debían examinar. Se les tomó muestra de sangre que se examinó mediante cuatro técnicas (examen en fresco, gota gruesa, prueba de Knott y conteo por c.c.), examen médico, estudio de saneamiento y encuesta por vivienda. Se encontró una prevalencia de $57 \%$ en el grupo examinado que, por ser muestra representativa de la población, se puede decir que es la prevalencia de la zona. Se tomó como unidad de análisis a 37 viviendas escogidas aleatoriamente. Existe una relación dada por la razón de prevalencia entre la presencia de la enfermedad con la ocupación de ser agricultor y síntomas como cefalea, dolor en el cuello, escalofríos y decaimiento, que fueron estadísticamente significativas. De las pruebas diagnósticas utilizadas, además de la prueba de Knott, el conteo por cc fue la que mejor resultado brindó en el estudio. Las condiciones de saneamiento ambiental en la zona fueron muy similares y no permitieron detectar diferencias entre las comunidades estudiadas. Se trató de tomar muestras entomológicas para la identificación de vectores que no se pudieron identificar plenamente.
\end{abstract}

\section{Summary.}

In the Guainia department of Colombia a study in the form of a cross-sectional prevalency survey was carried out in seven communities along the Rio Atabapo, where it was calculated that 181 people older than 10 years of age should be examined, taking 37 randomly chosen dwellings as the cone analysis unit. Blood samples were taken and subjected to four analysis techniques (fresh blood analysis, thick smear, Knott test and content per $\mathrm{cc}$ ); medical examinations were given, health studies made and housing questionnaire carried out. A $57 \%$ prevalency was found in the group examined from which, being a representative sample of the population, it can be said that there it is the zone prevalence. Given the prevalence, a relationship exists between the presence of disease with the occupation of being a farmer and symptoms such as headache, neckache, shivering and weakness, which were statistically significant. Of the diagnostic tests used in addition to the Knott test, cc content was that which offered the best result in this study. Environmental health conditions in the zone were similar and did not allow detection of differences between the communities studied. Entomological samples were taken to identify vectors which it was not possible to fully identify.

\footnotetext{
1 Secretaría de Salud del Guainía, Puerto Inírida, Guainía.

${ }^{2}$ Banco de Sangre, Hospital Inírida, Puerto Inírida, Guainía.
} 
La mansonelosis es una enfermedad ocasionada por parásitos de un grupo de enfermedades llamado filarias, que se distribuyen en zonas tropicales y subtropicales; es causada por un nemátodo en forma de filamento que ocasiona diferentes lesiones en el humano, de acuerdo con la especie de filaria que lo afecte (1). Son transmitidas a través de dipteros hematógafos como Simulium, Culex, Mansonia, Tabanides y Culicoides (2- 4). Las filarias que tienen importancia médica son: Wuchereria bancrofti, Brugia malayi, Onchocerca volvulus, Loa loa, Ancathochelonema, Mansonella pertans y Mansonella ozzardi (1). En Colombia, se han detectado mansonelas en los departamentos de Casanare, Vichada, Meta, Vaupés y Guainía, en especial, M. ozzardi(5-11).

En el departamento del Guainía se han detectado mocrofilarias en pacientes que consultaron por presentar síntomas clínicos inespecíficos que se relacionaban con malaria y a quienes se les ordenó un examen de gota gruesa para el diagnóstico. Esto creó la inquietud de realizar un estudio que estableciera la prevalencia de mansonelosis en comunidades del Guainía; se escogieron las que se encuentran a lo largo del río Atabapo para describir las características de la enfermedad, las variables relacionadas con la enfermedad y evaluar las pruebas de laboratorio para su diagnóstico (12, 13).

\section{Materiales y métodos}

El departamento del Guainía se encuentra situado en el extremo centroriental de Colombia; tiene como límites: al norte, el departamento del Vichada; al oriente, la república de Venezuela; al sur, la república de Brasil y, al occidente, los departamentos de Guaviare y Vaupés. El departamento tiene una extensión geográfica de 72.238 $\mathrm{km}^{2}$; su relieve es poco ondulado aunque presenta algunas elevaciones como la Sierra del Naquén, con una altura promedio de $200 \mathrm{msnm}$.

Posee una riqueza hidrográfica abundante; los principales ríos son el Guaviare, el Inírida, el Guainía y el Atabapo, además de los incontables caños y ríos menores que forman en conjunto una amplia red fluvial en toda la extensión del territorio del departamento, la cual es la base del sistema de transporte y de comunicaciones.
El clima del departamento es tropical, cálido, con una temperatura media de $30^{\circ} \mathrm{C}$ y un elevado grado de humedad relativa $(70-90 \%)$, con variaciones climáticas de los períodos de lluvia que conforman las dos estaciones, la de invierno (abril-noviembre) y la de verano (diciembre-marzo). La vegetación de la región está conformada por bosques selváticos, zona de selva tropical húmeda y, en menor proporción, presenta áreas de sabana, especial-mente hacia el nororiente del departamento.

La zona escogida para el estudio comprendió las comunidades localizadas en la orilla del río Atabapo, frontera con Venezuela, 7 localidades que se encuentran entre la comunidad de Chaquita hasta la población de Raudal Pato, habitadas por indígenas de las etnias Curripaco, Baniva y Piapoco; el número de colonos en la zona es mínimo. Las comunidades más cercanas se encuentran a 6 horas por vía fluvial desde el municipio de Inírida (Chaquita) hasta 12 horas a la más lejana (Raudal Pato). Esta es una zona de tránsito entre el municipio de Inírida y la parte media del río Guainía o río Negro, siendo, además, área de colonización de grupos indígenas Curripacos, procedentes del río Guainía alto.

El estudio realizado fue una encuesta de prevalencia de corte donde a través de la toma de exámenes de laboratorio, del estudio de saneamiento, de la encuesta familiar y del examen médico individual se buscó establecer la prevalencia de mansonelosis, conocer algunas características de la enfermedad, su comportamiento y la magnitud de esta parasitosis en el área de investigación. Se trató de describir variables asociadas relacionadas con la transmisión de la enfermedad y realizar una evaluación de técnicas de los procesos de diagnóstico de laboratorio (1).

La vivienda se tomó como marco muestral y se escogieron mediante muestreo aleatorio simple por conteo de viviendas de cada comunidad y escogiéndolas despúes de levantar los planos básicos de cada localidad.

Para el cálculo de la muestra, se tomó como referencia la prevalencia de $25,8 \%$ para filariasis por M. ozzardi encontrada en el estudio realizado 
en la comisaría del Guainía por el grupo de W. Kozek y col. en 1981 (6) para todo el departamento, sin haber sido un estudio con una muestra poblacional representativa. Se calculó un error estándar de $5 \%$ y se utilizó la fórmula para poblaciones finitas; la muestra fue de 180 personas en un número aproximadamente de 37 viviendas (seis habitantes por vivienda) para la zona del río Atabapo.

\section{Resultados}

La distribución poblacional (cuadro 1) y por ocupación (figura 1) en el estudio mostró que la actividad predominante es la agricultura, $71 \%$, y la realiza un grupo lingüístico Curripaco con $72,9 \%$ (132), seguido por Banivas con 14,3\% (26) y Yerales 5,5\% (10); el número de hombres fue de $50,2 \%$ (91) y mujeres, $49,1 \%$ (89). El número de pacientes examinados fue muy similar. Las localidades donde se tomaron las muestras fueron: Cacahual, 29,3\% (53), Chaquita con 25,9\% (47) y la comunidad de Merey, 16,5\% (30); se examinó la población mayor de 10 años con excepción de dos pacientes de 9 y 8 años que eran positivos.

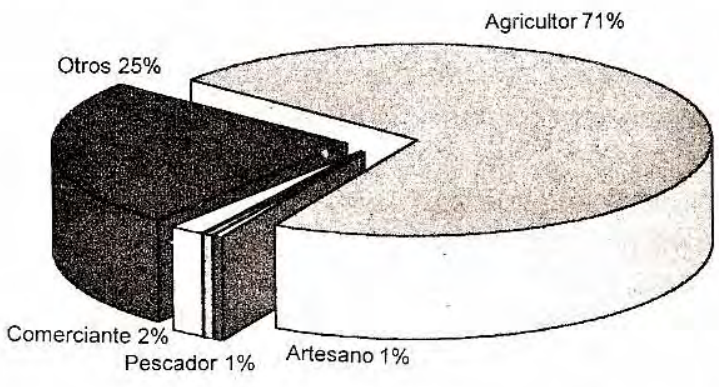

Figura 1. Ocupación predominante de los habitantes examinados.
Los síntomas referidos por las personas examinadas durante el estudio fueron: cefalea, fiebre, dolor del cuello, decaimiento y escalofríos (cuadro 2). El diagnóstico final de las personas examinadas fue: sanos, $58 \%$; pterigios, $32 \%$; embarazo, $2,8 \%$; rinofaringitis, $1,7 \%$, y otros con $5,5 \%$. No se detectaron durante el estudio casos de otras enfermedades transmitidas por vectores (malaria, leishmaniasis, etc.).

Los resultados de laboratorio mostraron una positividad para microfiliarisis de $57,5 \%$ (104) en la prueba de Knott, 42,5\% (75) en el examen de gota gruesa, $42 \%$ (76) en el examen en fresco y $61,3 \%$ (111) en el conteo por $\mathrm{mL}$ (cuadro 3 ).

Al relacionar los positivos en la prueba de Knott con la sintomatología referida, se encontró una razón de prevalencia de 1,46 para ser agricultor y positivo para mansonelosis la cual fue estadísticamente significativa.

Cuadro 2. Distribución porcentual de síntomas detectados en el examen médico.

\begin{tabular}{lcc}
\hline Síntomas & $\mathbf{n}$ & $\%$ \\
\hline Fiebre & 45 & 24,9 \\
Cefalea & 70 & 38,7 \\
Dolor en cuello & 39 & 21,5 \\
Escalofríos & 30 & 16,6 \\
Decaimiento & 32 & 17,7 \\
Pérdida del conocimiento & 3 & 1,7 \\
Problemas visuales & 1 & 0,6 \\
\hline
\end{tabular}

En los síntomas, la fiebre presentó una razón de prevalencia de 1,23 que no fue estadísticamente significativa, presentando un chi cuadrado de 2,08 $y$ un valor de $p=0,1494$. La cefalea, el dolor de cuello, los escalofríos y el decaimiento dieron una razón de prevalencia de1,47, 1,55, 1,35 y 1,55, respectiva-mente, siendo todos estos resultados

Cuadro 1. Distribución de la muestra por etnia, comunidad y sexo, 1996.

\begin{tabular}{|c|c|c|c|c|c|c|c|c|c|c|c|c|c|c|c|c|}
\hline \multirow{2}{*}{$\begin{array}{l}\text { Etnia } \\
\text { Comunidad }\end{array}$} & \multicolumn{2}{|c|}{ Baniva } & \multicolumn{2}{|c|}{ Colono } & \multicolumn{2}{|c|}{ Curripaco } & \multicolumn{2}{|c|}{ Guariquen } & \multicolumn{2}{|c|}{ Puinave } & \multicolumn{2}{|c|}{ Yeral } & \multicolumn{2}{|c|}{ Total } & \multirow[b]{2}{*}{ n } & \multirow[b]{2}{*}{$\%$} \\
\hline & $\mathrm{F}$ & M & $\mathbf{F}$ & M & $\mathrm{F}$ & M & $\mathrm{F}$ & M & $\mathrm{F}$ & M & $\mathrm{F}$ & M & $\mathbf{F}$ & $M$ & & \\
\hline Cacahual & 9 & 16 & 2 & 1 & 4 & 1 & 6 & 2 & 1 & 1 & 3 & 7 & 25 & 28 & 53 & 29,3 \\
\hline Caño Rayo & 0 & 0 & 0 & 0 & 5 & 4 & 0 & 0 & 0 & 0 & 0 & 0 & 5 & 4 & 9 & 4,9 \\
\hline Chaquita & 0 & 0 & 0 & 0 & 26 & 21 & 0 & 0 & 0 & 0 & 0 & 0 & 26 & 21 & 47 & 25,9 \\
\hline Merey & 0 & 0 & 0 & 0 & 12 & 18 & 0 & 0 & 0 & 0 & 0 & 0 & 12 & 18 & 30 & 16,5 \\
\hline Pintao & 0 & 0 & 0 & 0 & 2 & 0 & 0 & 0 & 0 & 0 & 0 & 0 & 2 & 0 & 2 & 1,1 \\
\hline Raudal Pato & 0 & 0 & 0 & 0 & 8 & 9 & 0 & 0 & 0 & 0 & 0 & 0 & 8 & 9 & 17 & 9,3 \\
\hline San Juan & 0 & 1 & 0 & 0 & 11 & 11 & 0 & 0 & 0 & 0 & 0 & 0 & 11 & 11 & 23 & 12,7 \\
\hline Total & 9 & 17 & 2 & 1 & 68 & 64 & 6 & 2 & 1 & 1 & 3 & 7 & 89 & 91 & 181 & 100 \\
\hline
\end{tabular}


Cuadro 3. Resultados de laboratorio de las muestras tomadas.

\begin{tabular}{lccccc}
\hline $\begin{array}{l}\text { Prueba de } \\
\text { laboratorio }\end{array}$ & \multicolumn{2}{c}{ Positiva } & \multicolumn{2}{c}{ Negativa } & Total \\
\hline $\mathbf{n}$ & $\%$ & $\mathbf{n}$ & $\%$ & \\
\hline Examen en fresco & 76 & 42,0 & 105 & 58,0 & 181 \\
Prueba de Knott & 104 & 57,5 & 77 & 42,5 & 181 \\
Gota gruesa & 75 & 42,5 & 103 & 57,9 & 181 \\
Conteo por cc & 111 & 61,3 & 70 & 38,7 & 181 \\
\hline
\end{tabular}

\begin{tabular}{|c|c|c|c|c|}
\hline A & & \multicolumn{2}{|c|}{ Prueba Knott } & \\
\hline$\stackrel{g}{r}$ & & + & - & Total \\
\hline $\begin{array}{l}\mathrm{i} \\
\mathrm{c} \\
\mathrm{u}\end{array}$ & + & 82 & 48 & 130 \\
\hline $\begin{array}{l}1 \\
t\end{array}$ & - & 22 & 29 & 51 \\
\hline $\begin{array}{l}0 \\
\mathrm{r}\end{array}$ & Total & 104 & 77 & 181 \\
\hline
\end{tabular}

\begin{tabular}{|c|c|c|c|c|}
\hline \multicolumn{5}{|c|}{ Prueba Knott } \\
\hline $\begin{array}{l}\mathrm{C} \\
\mathrm{e}\end{array}$ & & + & - & Total \\
\hline $\begin{array}{l}f \\
a\end{array}$ & + & 50 & 20 & 70 \\
\hline 1 & - & 54 & 57 & 111 \\
\hline a & Total & 104 & 77 & 181 \\
\hline \multicolumn{5}{|c|}{ Prueba Knott } \\
\hline & & + & - & Total \\
\hline 1 & + & 31 & 8 & 39 \\
\hline $\begin{array}{l}0 \\
r\end{array}$ & - & 73 & 69 & 142 \\
\hline & Total & 104 & 77 & 181 \\
\hline
\end{tabular}

\begin{tabular}{lcccr}
\hline & \multicolumn{4}{c}{ Prueba Knott } \\
E & & + & - & Total \\
$\mathrm{s}$ & + & 22 & 8 & 30 \\
$\mathrm{c}$ & - & 82 & 69 & 151 \\
$\mathrm{a}$ & - & 77 & 181 \\
$\mathrm{I}$. & Total & 104 & 77 \\
\hline
\end{tabular}

\begin{tabular}{rrrrr}
\hline \multicolumn{5}{c}{ Prueba Knott } \\
$\mathrm{D}$ & & + & - & Total \\
$\mathrm{e}$ & + & 26 & 6 & 32 \\
$\mathrm{c}$ & & 78 & 71 & 149 \\
$\mathrm{a}$ & - & 77 & 18 \\
$\mathrm{i}$ & Total & 104 & 77 \\
$\mathrm{~m}$. & & & \\
\hline
\end{tabular}

estadísticamente significativos. No fue posible relacionar los hallazgos físicos con la presencia de mansonela ya que a las personas examinadas no se les encontraron signos físicos relacionados con la enfermedad.

\section{Discusión}

El estudio se realizó en las comunidades del río Atabapo durante el mes de julio, época en la cual se presentan abundantes lluvias y son posibles las comunicaciones fluviales entre las diferentes comunidades investigadas. Durante el tiempo en que se realizó el recorrido, mucha de la población que vive en las comunidades no se encontraba ya que estaban en los lugares de cultivos (Conucos) y donde recogen la fibra de Chiqui-Chiqui. Por esta razón, la población real que se tomó para el estudio fue mucho menor (570) que la que se tuvo en cuenta para el cálculo de la muestra al iniciar la investigación (730). El número total de pacientes examinados (181) resultó ser una muestra poblacional suficiente para el estudio realizado.

\section{Variables generales}

Analizando los resultados de la prueba de Knott, tomada como la prueba de oro para el diagnóstico y por ser una de las que dió mayor positividad para la identificación de filaria del tipo $M$. ozzardi, se cruzó con las variables que presentaron resultados positivos en el examen médico, para así poder establecer con cierta aproximación una relación entre la presencia de parásitos, la sintomatología y los hallazgos clínicos encontrados. Se calcularon las razones de prevalencia de los positivos a filarias y los síntomas y signos encontrados.

Al relacionar los resultados de la prueba de Knott con la ocupación predominante de los estudiados, que fue la agricultura, se puede observar que la razón de prevalencia de ser agricultor y positivo para filaria es de 1,46, lo que muestra que se presenta estadísticamente una relación entre ser agricultor y presentar la positividad para la enfermedad.

Al cruzar los positivos con la prueba de Knott y la categoría de otros en ocupación predominante, se encontró una razón de prevalencia de 0,63. Este resultado es complementario e inverso al anterior ya que el ser agricultor y el tener otras actividades (escolares, hogar, maestro) son las que más refirieron las personas estudiadas (96\%). Además, se debe tener en cuenta que la edad es un factor de confusión debido a que los escolares, por su edad, han tenido menor tiempo de exposición a 
los vectores. Sin embargo, este dato muestra que existe una protección significativa para la presencia de la enfermedad. También puede ser debido a que la mayor parte de los que están en la categoría, son personas que no tienen que penetrar o permanecer continuamente en la selva dentro de sus actividades corrientes donde es probable que se encuentren los vectores transmisores de la enfermedad.

\section{Síntomas detectados en la historia clínica}

Al realizar los cálculos, la razón de prevalencia de los pacientes con prueba de Knott positiva y que presentaron fiebre, se dió el resultado que se inter-preta como si esta variable no se relacionara con los pacientes que fueron positivos para $M$. ozzardi, sin ser estadísticamente significativo.

Con la cefalea, los pacientes positivos a la prueba de Knott presentaron una razón de prevalencia de 1,47 ,estadísticamente significativa. Se interpreta como si la cefalea sí tuviera una relación importante con los pacientes que presentaron positividad en las pruebas Knott, siendo estadísticamente significativa.

Con relación al dolor de cuello, los pacientes positivos en la prueba de Knott presentaron una razón de prevalencia de 1,55. Se interpreta como si el dolor que se localiza en la parte posterior del cuello fuera significativo en los pacientes positivos a la mansonelosis.

Con relación al síntoma de escalofríos, se encontró una razón de prevalencia de 1,35 cuando los pacientes eran positivos. El escalofrío como síntoma tiene un significado estadístico apreciable, relacionado con los positivos de filariasis.

Cuando se relaciona el decaimiento con los pacientes positivos en la prueba de Knott, encontramos una razón de prevalencia de 1,55, estadísticamente significativa.

Estos síntomas que se relacionan en la razón de prevalencia con la positividad a la mansonelosis, son los mismos que refieren muchos de los pacientes que consultan en las unidades de salud del departamento y que resultan afectados por estas parasitosis. Algunos de estos síntomas también fueron comentados en el estudio realizado en Chocó durante 1993(13).

Al evaluar los otros síntomas y signos interrogados durante el examen físico de los pacientes a los que se les tomó muestra de laboratorio (pérdida de conocimiento, problemas visuales, masas, edemas etc.) no es posible correlacionarlos con la positividad a mansonelosis porque los evaluados no refirieron presencia de estos síntomas o signos.

En el examen físico, se encontró positiva la presencia de pterigios de diferentes grados y la mayoría bilaterales en 69 de los examinados. En la evaluación de fondo de ojo, se encontró opacidad del cristalino en diferentes grados en 19 de los evaluados. En la literatura médica no se comenta que las filarias y, en especial, $M$. ozzardi causen lesiones oculares de algún tipo, con excepción de 0 . volvulus $(1,14,15)$.

Al analizar las otras variables, no se puede relacionar con la positividad a filarias, ya que no se evidenciaron lesiones o signos apreciables durante el examen físico.

\section{Conclusiones}

Después de analizados los diferentes aspectos de la investigación, se pudo llegar a las siguientes conclusiones.

La prevalencia de filaria en la zona estudiada del río Atabapo fue de $57,5 \%$ en el grupo de personas examinadas, donde se pudo establecer que eran M. ozzardi. Teniendo en cuenta que la muestra es representativa para la población de toda esta zona, podemos afirmar que la filaria presenta una alta prevalencia en las personas que viven en esta región. Si se tiene en cuenta estudios anteriores como el de W. Kozek (6-9) donde se encontró una prevalencia de $25,8 \%$ para esta mansonelosis, se puede concluir que la enfermedad ha presentado un aumento considerable en la zona.

Los grupos humanos más afectados fueron las personas entre los 20 y 40 años, población económicamente activa, en su gran mayoría agricultores ( $71,8 \%)$, en especial en recolección de fibra de Chiqui-Chiqui y cultivos de pancoger y que se desplazan de sus comunidades a los sitios de cultivo (conucos) en promedio de 3 a 6 horas. 
La presencia de $M$. ozzardi se presentó sin una gran diferencia entre hombres y mujeres, donde el grupo indígena con mayor predominio en la positividad a la enfermedad fue el grupo étnico de los Curripacos, los cuales son mayoría en la zona del río Atabapo.

Al relacionar las diferentes variables positivas en el grupo estudiado con la positividad de la enfermedad dada la prueba de Knott, encontramos lo siguiente: la actividad agrícola presentó una relación positiva estadísticamente significativa (razón de prevalencia de 1,46\%), relacionar las otras actividades (estudiante, ama de casa) se encontró una relación protectora con una razón de prevalencia de 0,63 , estadísticamente significativa. Esto se puede deber a que la transmisión de la enfermedad es posible que no se lleve a cabo en el área urbana de cada localidad sino en la zona de cultivos de la región selvática. También, se puede ver que la mayoría de los que presentan otro tipo de actividades eran menores de 15 años, por lo cual es una variable de confusión para este análisis.

Al relacionar la positividad con los que presentan fiebre, se evidenció una asociación en los pacientes estudiados, dándose razones de prevalencia que no fueron estadísticamente significativas. Con la cefalea, el decaimiento, el escalofrío y el dolor de cuello, se observaron razones de prevalencia altas que tienen un significado estadístico.

Se utilizaron cuatro técnicas de laboratorio para el diagnóstico donde la prueba de Knott se tomó como la prueba de oro. Al relacionar las diferentes prue-bas utilizadas en el estudio con la prueba de oro, se pudo establecer que el examen más fiable era el conteo por centímetro cúbico ya que mostró la mejor sensibilidad, especificidad, valor predictivo positivo y valor predictivo negativo, en relación con el examen en fresco y la gota gruesa.

Las comunidades visitadas presentan características de vivienda, de saneamiento ambiental, de cultura y de procesos económicos muy similares entre ellas. Son comunidades sedentarias donde sus habitantes se desplazan a la zona selvática para desarrollar actividades de agricultura como la recolección de fibra de Chiqui-
Chiqui y cultivos para consumo. El desarrollo de saneamiento ambiental es escaso; el río y los caños forman parte impor-tante de su desarrollo social y ambiental. Por ser estas comunidades tan homogéneas, no fue posible establecer una relación de las variables de vivienda y saneamiento básico con la presencia de mansonelosis en las comunidades.

En las localidades visitadas no se encontraron criaderos positivos abundantes de posibles vectores en sus cercanías y en la zona no se observó la presencia de otras enfermedades transmitidas por vectores (malaria, leishmaniasis) a pesar de que existe presencia de posibles vectores $(2,16)$.

La zona del río Atabapo es de comunicación entre los ríos Inírida, Guaviare y Orinoco con la parte media del río Guainía, área donde se ha detectado prevalencia de filarias del tipo $M$. ozzardien las comunidades Curripacas (12), grupos humanos que han colonizado el río Atabapo, pudiendo ser una ruta por donde se está desplazando la enfermedad a otras áreas del departamento.

La mansonelosis por $M$. ozzardi en el área del río Atabapo se convierte en la enfermedad transmitida por vectores de mayor prevalencia en la zona y que afecta a un gran número de personas de forma variable y por determinar.

\section{Recomendaciones}

Se hace necesario continuar con el estudio de mansonelosis en la zona del río Atabapo y en otras áreas del Guainía, debido a la alta prevalencia de esta parasitosis. Los estudios que se realicen en el futuro deberán profundizar y establecer la relación de la enfermedad con los síntomas que se encontraron como cefalea, dolor de cuello y decaimiento.

Para establecer programas de control, es necesario establecer los posibles vectores de $M$. ozzardi mediante un estudio entomológico en la zona del río Atabapo.

Se debe iniciar un plan de tratamiento a las personas afectadas por $M$. ozzardi, donde se estudie la efectividad, la seguridad y la disminución de la presencia del parásito en los pacientes, utilizando los medicamentos recomendados por 
la literatura (17). Se podría comparar la eficacia de varios medicamentos como la ivermectina, el levamizol y el albendazol.

Se deben estudiar los hábitos de los vectores mediante el estudio de la periodicidad de las microfilarias en sangre, para así poder llegar a establecer si en esta zona $M$. ozzardi presenta algún ciclo o realmente no tiene ninguno como lo menciona la literatura médica mundial $(1,4)$.

Diseñar programas con la participación de las comunidades, que estén orientados a lograr el mejoramiento del saneamiento básico en cada una de las diferentes localidades ya que la mayoría presentan grandes deficiencias.

Establecer un programa de atención oftalmológica en la zona para dar tratamiento adecuado a las lesiones oculares encontradas, pterigios y opacidad del cristalino, tratando de establecer las causas reales de la presencia de estas lesiones en los habitantes de esta zona.

Se recomienda continuar con los estudios de filaria y especialmente de Mansonella en otras áreas del departamento, como en el medio y bajo río Negro o río Guainía.

Aplicar como prueba de laboratorio de campo para el diagnóstico de microfiliarias en sangre, la técnica de conteo por $\mathrm{mL}$ que mostró una sensibilidad y especificidad similar a la prueba de oro (técnica de Knott).

\section{Referencias}

1. Botero D, Restrepo M. Parasitología humana. Medellín: Corporación para Investigacioners Biológicas; 1987:134-44.

2. Tidwell MA, Tidwell M, Muñoz $\mathbf{P}$. Vectores de Onchocerca volvulus y Mansonella ozzardi en Colombia. Acta Médica 1980;11(4):119-27.

3. Barreto P, Trapido H. Onchocerciasis in Colombia: entomological findings in the first observed focus. Am J Trop Med Hyg 1970;19(5):837-41.
4. Muñoz de Hoyos P. Simúlidos del río Chisacá. Bogotá: Facultad de Ciencias, Universidad Ncional de Colombia, 1990.

5. Kosek W, D'Alessandro J. Filariasis en Colombia: prevalencia de mansonelosis en la población joven y adulta de la orilla colombiana del río Amazonas, comisaría del Amazonas. Colombia Médica 1984; 15(1):35-40.

6. Kosek W, Palma G, et al. Filariasis en Colombia y distribución de Mansonella ozzardi y Mansonella perstans en la comisaría del Guainía. Acta Médica, 1985;16(3-4):137-42.

7. Kosek W, D Alessandro J. Filariasis en Colombia: presencia de Dipetalomena perstans en la comisaría del Guainía. Acta Médica 1983;14(4):1736.

8. Corredor A. Ampliación de la distribución geográfica de Mansonella ozzardi en la República de Colombia. Rev Fac Med UN 1963;3:3-5.

9. Kosek WJ, Palma G, et al. Filariasis en Colombia: prevalencia de Mansonella ozzardi en el departamento del Meta, intendencia del Casanare y comisaría del Vichada. Acta Médica 1986;17(1):402.

10. Orihel TC. Filariasis humana en el Nuevo Mundo. Acta Médica 1982;13(4):119-22.

11. Marinkelle CJ. Filarisis en Colombia. Acta Médica 1982;13(4):123-5.

12. Chiquito M, Cueto V. Informes de bacteriología de la comisión multidisciplinaria al río Guainía, 1995.

13. Padilla JC. Prevalencia de filarias en el departamento del Chocó: informe preliminar, SEM, regional Chocó, 1993 (datos no publicados).

14. Beaver PC. Clínica y complicaciones de las parasitosis; 1986:383-401.

15. Little MD, Assis-Masri G. A case of ocular onchocercosis in Colombia. Roy Soc Trop Med Hyg 1965;59(6):717.

16. CIDEIM. Manual de entomología médica para investigadores de América Latina. Cali; 1993.

17. Godman y Gilman. Las bases farmacológicas de la terapéutica. Séptima edición. Editorial Médica Panamericana; 1980. 